\title{
Temperature dependent characteristics of step-edge YBCO SQUIDs
}

\author{
H.E. Horng ${ }^{a}$, S.Y. Yang ${ }^{a}$, J.T. Jeng ${ }^{a}$, and H.C. Yang
}

${ }^{a}$ Department of Physics, National Taiwan Normal University, Taipei 116, Taiwan

bepartment of Physics, National Taiwan University, Taipei 106, Taiwan

In this report, the temperature dependent characteristics are investigated for the step-edge YBCO SQUID. The experimental results show a linear relationship between $I_{c}$ and $T$. This reveals a SIS-like tunneling behavior for the step-edge YBCO SQUID. The peak-to-peak voltage $V_{p p}$ in the V- $\Phi$ curve of the SQUID was found to decrease almost linearly when the temperature was raised from $10 \mathrm{~K}$ to $60 \mathrm{~K}$. As the temperature approached to the $T_{c}$ of the SQUID, the $V_{p p}$ was reduced abruptly and deviated from the theoretically predicted curve. This deviation may be due to the variation of the inductance of the SQUID at various temperatures.

\section{INTRODUCTION}

Due to their great potential in applications, the characteristics of high- $T_{c}$ SQUIDs have received increasing studies [1-6]. Most reports have focused on optimization of the SQUIDs and some significant results were obtained $[1,2,6]$. However, research on the temperature effects on the SQUID characteristics are still rare. Therefore, some properties of the stepedge YBCO SQUID are investigated at various temperatures in this work.

\section{EXPERIMENMTAL DETAILS}

The SQUID investigated is a step-edge YBCO SQUID with a hole area of $20 \times 40 \mu \mathrm{m}^{2}$. The fabrication of the SQUID was reported elsewhere [5]. For the electric measurements, the four-point method was used. The temperature was sensed by a diode thermometer and controlled by a temperature controller. To examine the magnetically modulated voltage oscillation of the SQUID, the SQUID was placed into a solenoid which provided a magnetic field perpendicular to the hole of the SQUID. The experimental details were illustrated in Ref. [6].

\section{RESULTS AND DISCUSSION}

The critical current $I_{c}$ of the step-edge YBCO This work is supported by the National Science Council under grand Nos. 89-2112-M-003-012 and 89-2112-M-002-028.
SQUID was obtained from the V-I curve with a criterion voltage of $1 \mu \mathrm{V}$. By varying the temperature, the $I_{c}$ as a function of the temperature $T$ can be examined. The experimental temperature dependent normalized critical current $I_{c}(T) / I_{c}(0)$ is shown in Fig. 1 (denoted by dots). It was found that the critical current $I_{c}$ almost remains constant at temperature $\mathrm{T}$ lower than $20 \mathrm{~K}$ When the temperature is raised, $I_{c}$ is reduced linearly down to zero as $T$ reaches to the critical temperature. This linearity suggests that the weak link of the step-edge YBCO junctions is similar to a SIS junction behavior. A theoretical $I_{c} / I_{c}(0)$-T relation for an ideal SIS Josephson junction was developed by Ambergokar-Baratoff to be [7]

$\mathrm{I}_{\mathrm{c}}(\mathrm{T}) / \mathrm{I}_{\mathrm{c}}(0)=[\Delta(\mathrm{T}) / \Delta(0)] \tanh \left(\alpha \mathrm{T}_{\mathrm{c}} / 2 \mathrm{~T}\right)$

here $\alpha$ is $\Delta(0) / k_{B} T_{c}, k_{B}$ is the Boltzmann constant

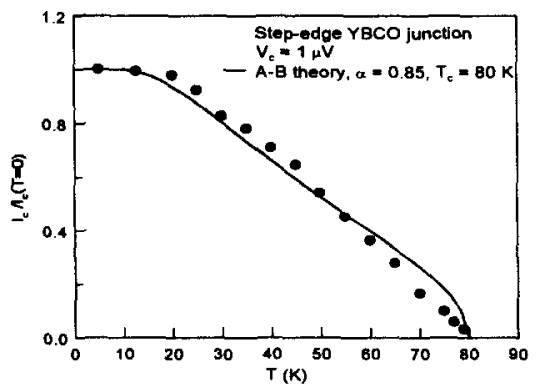

Fig. 1. Normalized critical current versus the temperature. The dots are the experimental data. The solid line is the theoretical curve [7] 
and $\Delta(T)$ is the temperature dependent energy gap in the BCS theory. To fit the experimental results to the theoretical curve, the $\Delta(T) / \Delta(0)$ can be evaluated at the reduced temperature $T / T_{c}$ with the help of the BCS functions tabulated in Ref. [8]. The theoretical curve with $\alpha=0.85$ and $T_{c}=80 \mathrm{~K}$ is shown in Fig. 1 by the solid line. It was found that the experimental data almost follow the SIS-junction behavior. And also, with the fitted $\alpha\left(=\Delta(0) / k_{B} T_{c}\right)$ being 0.85 and $T_{c}$ being $80 \mathrm{~K}$, the $\Delta(0)$ was obtained to be $5.82 \mathrm{meV}$.

In addition to the temperature dependent critical current of the SQUID, the influence of the temperature on the magnetically modulated voltage oscillation (V- $\Phi$ curve) is also investigated. In Fig. 2 , the peak-to peak voltage $V_{p p}$ of the $V-\Phi$ curve decreased almost linearly when the temperature was increased from $10 \mathrm{~K}$ to $60 \mathrm{~K}$. An abrupt drop in the $V_{p p}$ was found at the temperatures from $60 \mathrm{~K}$ to $77 \mathrm{~K}$. Based on the RSJ model with thermal fluctuations for a SQUID, Enpuku derived a theoretical expression for the $V_{p p}$ as [9]

$V_{P P}=v \frac{I c R n}{1+\beta_{L}}\left(1-3.57 \frac{\sqrt{k_{B} T L}}{\Phi_{0}}\right)$

with $\beta_{\mathrm{L}}=\mathrm{LI}_{\mathrm{c}} / \Phi_{\mathrm{o}}$, where $\nu$ is a bias current dependent variable, $I_{c}$ is the critical current of the SQUID without thermal fluctuations, $R_{n}$ is the normal state resistance of the SQUID, $k_{B}$ is the Boltzmann constant, $T$ is the working temperature and $L$ is the inductance of the SQUID. For this case, the $R_{n}$ equals $6 \Omega$. The range of the working temperature is from $10 \mathrm{~K}$ to $77 \mathrm{~K}$ and the $I_{c}$ at each temperature is obtained from the V-I curve. In the experiment, the bias current for the maximum $V_{p p}$ at a given temperature almost equals to the

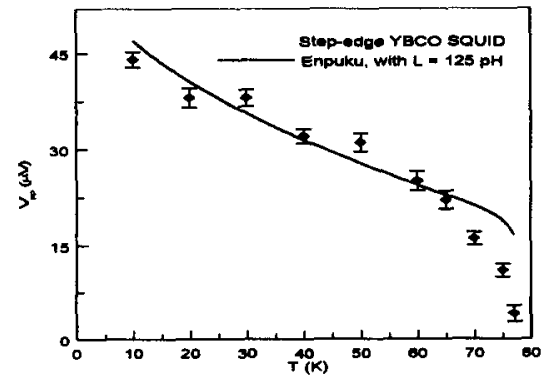

Fig. 2. $V_{p p}$ in the $V-\Phi$ curve of the SQUID as a function of temperature. The dots with error bars are data points, whereas the line was deviated by Enpuku et al. [9]. corresponding $\mathrm{I}_{\boldsymbol{c}}$. According to the published report [10], the suitable value for $v$ is $7 / \pi^{2}$. The only undetermined parameter in Eq. (2) is L. To find it, the experimental data $V_{p p}(T)$ are fitted to Eq. (2). The resulting curve with $\mathrm{L}=125 \mathrm{pH}$ is shown in Fig. 2 (denoted by the solid line). A good match between the experimental data and the theoretical prediction is obtained at temperatures lower than 60 $\mathrm{K}$. Whereas an obvious deviation of the data from the fitted curve occurs at higher temperatures. This deviation may be due to the variation in the inductance $\mathrm{L}$ at higher temperature. This point is also supported by the published researches from other groups $[11,12]$.

\section{CONCLUSION}

The $I_{c}-T$ curve reveals SIS-like tunneling behavior for the step-edge YBCO SQUID. The $V_{p p}$ in the $V-\Phi$ curve of the SQUID decreased with increasing the temperature. The $\mathrm{V}_{\mathrm{pp}}-\mathrm{T}$ curve is consistent with the theoretical prediction in the lower temperature range. At high temperatures, the deviation of the experimental $\mathrm{V}_{\mathrm{pp}}-\mathrm{T}$ curve from the theoretical prediction implies that the inductance of the SQUID is heavily temperature dependent at temperatures near $T_{c}$.

\section{REFERENCES}

1. D. Drung, R. Contor, M. Peters, H.J. Scheer, and H. Koch, Appl. Phys. Lett., 57 (1990) 406.

2. H.C. Yang, J.H. Lu, S.D. Lin, L.C. Ku, H.M. Cho, W.B. Jian, M.Y. Chen, H.E. Horng, J.M. Wu, J.T. Lo, and C.C. Chiou, J. Appl. Phys., 78 (1995) 1871.

3. A.H. Miklich, D. Koelle, F. Ludwig, D.T. Nemeth, E. Dantsker, and J.Clarke, Appl. Phys. Lett., 66 (1995) 230.

4. R. Weidl, S. Brabetz, F. Schmidl, S. Wunderlich, and P. Seidel, Supercond. Sci. Technol., 10 (1997) 95.

5. T.S Lee, Y.R. Chemla, E. Dantskar, and J.Clarke, IEEE Trans. Appl. Supercond., 7 (1997) 3147.

6. S.Y. Yang, C.H Chen, H.E Horng, W.L. Lee, and H.C. Yang, IEEE Trans. Appl. Supercond., 9 (1999) 3121.

7. V. Amegaokar and A. Baratoff, Phys. Rev. Lett., 10 (1963) 486.

8. B. Muhlschlegel, Z. Phys., 155 (1959) 313.

9. K. Enpuku, Y. Shimomura, and T. Kisu, J. Appl. Phys., 73 (1993) 7929.

10. T. Ryhanen, H. Seppa, R.Ilmoniemi, and J. Kanuutila, J. Low Temp. Phys., 76 (1989) 287.

11. G. Hildebrandt and F.H. Uhlmann, IEEE Trans. Appl. Supercond., 5 (1995) 2766.

12. V.K. Kaplunenko et al., IEEE Trans. Appl. Supercond., 5 (1995) 2835. 